\title{
$D$-functions and immanants of unitary matrices and submatrices
}

\author{
Hubert de Guise ${ }^{1}$, Dylan Spivak ${ }^{1}$, Justin Kulp ${ }^{1}$ and Ish Dhand ${ }^{2}$ \\ ${ }^{1}$ Department of Physics, Lakehead University, Thunder Bay, Ontario P7B 5E1, \\ Canada \\ ${ }^{2}$ Institute for Quantum Science and Technology, University of Calgary, Calgary, \\ Alberta T2N 1N4, Canada
}

23 March 2022

\begin{abstract}
Motivated by recent results in multiphoton interferometry, we expand a result of Kostant on immanants of an arbitrary $m \times m$ unitary matrix $T \in \mathrm{SU}(m)$ to the submatrices of $T$. Specifically, we show that immanants of principal submatrices of a unitary matrix $T$ are a sum $\sum_{t} D_{t t}^{(\lambda)}(\Omega)$ of the diagonal $D$-functions of group element $\Omega$, with $t$ determined by the choice of submatrix, and the irrep $(\lambda)$ determined by the immanant under consideration. We also provide evidence that this result extends to some submatrices that are not principal diagonal, and we discuss how this result can be extended to cases where $T$ carries an $\mathrm{SU}(m)$ representation that is different from the defining representation.
\end{abstract}

\section{Introduction and basic result}

The immanant $\operatorname{Imm}^{\{\tau\}}(T)$ of the matrix $m \times m$ matrix $T$, associated with the partition $\{\tau\}$ of $S_{m}$, is defined in [1] by

$$
\begin{aligned}
\operatorname{Imm}^{\{\tau\}}(T) & :=\sum_{\sigma} \chi^{\{\tau\}}(\sigma) P(\sigma)\left[T_{11} T_{22} \ldots T_{m m}\right] \\
& \equiv \sum_{\sigma} \chi^{\{\tau\}}(\sigma) T_{1 \sigma(1)} T_{2 \sigma(2)} \ldots T_{m \sigma(m)},
\end{aligned}
$$

where $\sigma \in S_{m}$ permutes $k$ to $\sigma(k)$, and $\chi^{\{\tau\}}(\sigma)$ is the character of $\sigma$ in the irrep $\{\tau\}$ of $S_{m}$. In this paper we explore the connection between immanants and group functions (or $D$-functions) for the unitary groups, and extend a result of Kostant [2] to submatrices of the fundamental representation of these groups.

Our work is motivated in part by intense renewed interest in immanants of submatrices of unitary matrices and, in particular, of permanants [3] of submatrices and their use in multiphoton interferometry [4-8]. Thus, our results are interesting within the paradigm of the BosonSampling problem $[9,10]$ and its deep link to issues in computational complexity theory. 
Immanants of totally non-negative and of Hermitian matrices have been studied in [11-13]; our results instead are applicable to unitary matrices and depend on the wellknown duality between representations of the unitary and of the symmetric groups [14, $15]$.

This duality identifies irreps of $\mathrm{SU}(m)$ with irreps of $S_{N}$ with $N \leq m$. If $\{\lambda\}=\left\{\lambda_{1}, \lambda_{2}, \ldots, \lambda_{N}\right\}$ is a partition of $\lambda=\sum_{k} \lambda_{k}$ labelling an irrep of $S_{N}$, we choose to label irreps of $\mathrm{SU}(m)$ using the round backets $(\lambda)$ with $m-1$ entries defined by $\left(\lambda_{1}-\lambda_{2}, \ldots, \lambda_{N-1}-\lambda_{N}\right)$, and trailing zeroes omitted. Thus, the irrep $\{21\}$ of $S_{3}$ corresponds to the SU(4) irrep $(110) \sim(11)$, the SU(5) irrep (1100) (11) etc.

Group functions (or Wigner $D$-functions) are defined as the overlap between two basis states of the same irrep of SU $(m)$, one of which has been transformed by an element $\Omega \in \mathrm{SU}(m)$. If $\left|\psi_{\ell}^{(\lambda)}\right\rangle,\left|\psi_{t}^{(\tau)}\right\rangle$ are any two basis states in irreps $(\lambda)$ and $(\tau)$ respectively and $T^{(\lambda)}(\Omega)$ is the matrix representing element $\Omega \in \mathrm{SU}(m)$ in the irrep $(\lambda)$, then

$$
D_{\ell t}^{(\lambda)}(\Omega):=\left\langle\psi_{\ell}^{(\lambda)}\left|T^{(\lambda)}(\Omega)\right| \psi_{t}^{(\tau)}\right\rangle \delta_{\lambda, \tau} .
$$

Kostant [2] has shown a simple connection between immanants (defined in Eq. (1)) of the fundamental representation $T$ of $\mathrm{SU}(m)$ group elements and group functions $D_{t t}^{(\lambda)}$ of $\mathrm{SU}(m)$ with $t$ running over each of the zero-weight states in irrep $(\lambda)$. Specifically, let $\Omega \in \mathrm{SU}(m)$ and $T(\Omega)$ (no superscript) be the defining $m \times m$ representation of $\Omega$. Further define the matrix $\mathcal{D}^{(\tau)}(\Omega)$ by

$$
\left(\mathcal{D}^{(\tau)}(\Omega)\right)_{r t}=D_{r t}^{(\tau)}(\Omega)
$$

with $r, t$ restricted to labelling zero-weight states in the irrep $(\tau)$. Then we have [2]

$$
\operatorname{Imm}^{\{\tau\}}(T(\Omega))=\operatorname{Tr}\left[\mathcal{D}^{(\tau)}(\Omega)\right] .
$$

For $\mathrm{SU}(2)$, this result simply states that the permanent of the matrix

$$
T(\Omega)=\left(\begin{array}{cc}
\mathrm{e}^{-\left(\frac{1}{2}\right) i(\alpha+\gamma)} \cos \left(\frac{\beta}{2}\right) & -\mathrm{e}^{-\left(\frac{1}{2}\right) i(\alpha-\gamma)} \sin \left(\frac{\beta}{2}\right) \\
\mathrm{e}^{\left(\frac{1}{2}\right) i(\alpha-\gamma)} \sin \left(\frac{\beta}{2}\right) & \mathrm{e}^{\left(\frac{1}{2}\right) i(\alpha+\gamma)} \cos \left(\frac{\beta}{2}\right)
\end{array}\right),
$$

where $\Omega=(\alpha, \beta, \gamma) \in \mathrm{SU}(2)$ is the $\mathrm{SU}(2)$ function $\operatorname{Imm}^{\{2\}}(T(\Omega))=D_{00}^{1}(\alpha, \beta, \gamma)=\cos \beta$ while the determinant $\operatorname{Imm}^{\{1,1\}}(T(\Omega))=D_{00}^{0}(\alpha, \beta, \gamma)=1$. The trace of Eq. (4) contains a single term in both $\mathrm{SU}(2)$ cases as the zero-weight subspaces in irreps $J=1$ and $J=0$ are both one-dimensional. Here and henceforth we follow the physics notation of labelling $\mathrm{SU}(2)$ irreps using the angular momentum label $J=\frac{1}{2} \lambda$, such that $2 J$ is an integer. Thus, $D_{00}^{1}(\Omega)$ is an $\mathrm{SU}(2) D$-function in the three-dimensional irrep $J=1$.

\section{Notational details}

We first introduce a basis for $\mathbb{H}_{p}^{(1)}$, which is the $p$ 'th copy of the carrier space for fundamental irrep $\{1\} \equiv(1)$ of $\mathrm{SU}(m)$. We write this basis in terms of harmonic oscillator states according to

$$
\mathbb{H}_{p}^{(1)}=\operatorname{Span}\left\{a_{k}^{\dagger}\left(\omega_{p}\right)|0\rangle, k=1, \ldots, m\right\} .
$$


The label $\omega_{p}$ can be thought of as an internal degree of freedom, say the frequency, of the $p$ 'th oscillator. We introduce the (reducible) Hilbert space $\mathbb{H}^{(N)}:=\mathbb{H}_{1}^{(1)} \otimes \mathbb{H}_{2}^{(1)} \ldots \otimes \mathbb{H}_{N}^{(1)}$, which is spanned by the set of harmonic oscillator states of the type

$$
a_{k}^{\dagger}\left(\omega_{1}\right) a_{r}^{\dagger}\left(\omega_{2}\right) \ldots a_{s}^{\dagger}\left(\omega_{N}\right)|0\rangle, \quad k=1, \ldots, m ; \quad r=1, \ldots, m, \text { etc }
$$

Another ingredient we need is the action of the permutation group $S_{N}$ on $\mathbb{H}^{(N)}$. The action of $P(\sigma)$ is defined as

$$
\begin{aligned}
P(\sigma) a_{k}^{\dagger}\left(\omega_{1}\right) & a_{r}^{\dagger}\left(\omega_{2}\right) \ldots a_{s}^{\dagger}\left(\omega_{N}\right)|0\rangle \\
& =a_{k}^{\dagger}\left(\omega_{\sigma^{-1}(1)}\right) a_{r}^{\dagger}\left(\omega_{\sigma^{-1}(2)}\right) \ldots a_{s}^{\dagger}\left(\omega_{\sigma^{-1}(N)}\right)|0\rangle .
\end{aligned}
$$

Alternatively, one may consider each of the sets $\left\{a_{k}^{\dagger}\left(\omega_{p}\right) ; k=1, \ldots, m\right\}$, which are labelled by $p$, as a tensor operator that carries the defining irrep (1) of $\mathrm{SU}(m) .|0\rangle$ is invariant under the action of $S_{N}$ and $\mathrm{SU}(m)$ elements.

The algebra $\mathrm{u}(m)$ is spanned by the $S_{N}$-invariant operators

$$
\hat{C}_{i j}=\sum_{k=1}^{N} a_{i}^{\dagger}\left(\omega_{k}\right) a_{j}\left(\omega_{k}\right) \quad i, j=1, \ldots, m .
$$

The $\mathrm{su}(m)$ subalgebra is obtained from $\mathrm{u}(m)$ by removing the diagonal operator $\sum_{i=1}^{m} \hat{C}_{i i}$, so the Cartan subalgebra of $\mathrm{su}(m)$ is spanned by the traceless diagonal operators

$$
\hat{h}_{i}:=\hat{C}_{i i}-\hat{C}_{i+1, i+1}, \quad i=1, \ldots, m-1 .
$$

A basis for the irrep $(\lambda)$ of $\mathrm{SU}(m)$ is given in terms of the harmonic oscillator occupation number $n$ according to

$$
\left|\psi^{(\lambda)} n ; \Lambda\right\rangle=\left|(\lambda) n_{1} n_{2}, \ldots, n_{m} ;\left(\lambda^{\prime}\right) \ldots(J)\right\rangle,
$$

where $n:=\left(n_{1}, n_{2}, \ldots, n_{m}\right)$ and $n_{k}$ indicates the number of excitations in mode $k \leq m$. The weight of this state is equals the $(m-1)$-tuple $\left[n_{1}-n_{2}, n_{2}-n_{3}, \ldots, n_{m-1}-n_{m}\right]$. Finally, the multi-index $\Lambda:=\left(\lambda^{\prime}\right) \ldots(J)$ refers to a collection of indices, each of which labels irreps in the subalgebra chain

$$
\begin{array}{cccccc}
\mathrm{su}(m) & \supset & \mathrm{su}(m-1) & \supset & \cdots & \supset \mathrm{su}(2) \\
(\lambda) & & \left(\lambda^{\prime}\right) & & & (J)
\end{array}
$$

and is needed to fully distinguish states having the same weight. The representation labels are all integers, except as mentioned above for the half-integered $\mathrm{SU}(2)$ label $J$. We take the subalgebra $\mathrm{su}(k-1) \subset \mathrm{su}(k)$ to be spanned by the subset of the $k \times k$ hermitian traceless matrices of the form

$$
\left(\begin{array}{cccc}
0 & 0 & \ldots & 0 \\
0 & * & * & * \\
\vdots & * & * & * \\
0 & * & * & *
\end{array}\right)
$$

where $*$ denote possible non-zero entries in $\mathrm{su}(k-1)$. 
Using this notation, we can write the matrix representation of $\Omega \in \mathrm{SU}(3)$ in the fundamental representation (which is denoted by (1) once the trailing 0 has been eliminated) of $\mathrm{SU}(3)$ as

$$
T(\Omega)=\left(\begin{array}{lll}
D_{100(0) ; 100(0)}^{(1)}(\Omega) & D_{100(0) ; 010\left(\frac{1}{2}\right)}^{(1)}(\Omega) & D_{100(0) ; 001\left(\frac{1}{2}\right)}^{(1)}(\Omega) \\
D_{010\left(\frac{1}{2}\right) ; 100(0)}^{(1)}(\Omega) & D_{010\left(\frac{1}{2}\right) ; 010\left(\frac{1}{2}\right)}^{(1)}(\Omega) & D_{010\left(\frac{1}{2}\right) ; 001\left(\frac{1}{2}\right)}^{(1)}(\Omega) \\
D_{001\left(\frac{1}{2}\right) ; 100(0)}^{(1)}(\Omega) & D_{001\left(\frac{1}{2}\right) ; 010\left(\frac{1}{2}\right)}^{(1)}(\Omega) & D_{001\left(\frac{1}{2}\right) ; 001\left(\frac{1}{2}\right)}^{(1)}(\Omega)
\end{array}\right)
$$

with $\Omega \in \mathrm{SU}(3)$. The result of Kostant [2] applied to $\mathrm{SU}(3)$ then states that

$$
\begin{aligned}
\operatorname{Per}(T(\Omega))= & \operatorname{Imm}^{\{3\}}(T(\Omega))=D_{111(1) ; 111(1)}^{(3)}(\Omega), \\
& \operatorname{Imm}^{\{21\}}(T(\Omega))=D_{111(1) ; 111(1)}^{(11)}(\Omega)+D_{111(0) ; 111(0)}^{(11)}(\Omega), \\
\operatorname{Det}(T(\Omega))= & \operatorname{Imm}^{\{111\}}(T(\Omega))=D_{000(0) ; 000(0)}^{(0)}(\Omega)=1,
\end{aligned}
$$

For convenience, we lighten notation and use the symbols $T$ and $\Omega$ to respectively denote matrices and elements in different $\mathrm{SU}(m)$ without indicating $m$; this does not affect our conclusions as our results apply to any $m$.

The novel contribution of this paper is to extend result of [2] encapsulated in Eq. (4) to submatrices of the fundamental representations. In addition, using outer plethysms, we can extend the theorem to immanants of matrix representations of $\mathrm{SU}(m)$ beyond the fundamental representation and to matrix representations of subgroups of $\mathrm{SU}(m)$.

\section{Proving the theorem: the case $N=m$}

We consider the state $\left|\Psi_{123 \ldots m}\right\rangle=a_{1}^{\dagger}\left(\omega_{1}\right) a_{2}^{\dagger}\left(\omega_{2}\right) \ldots a_{m}^{\dagger}\left(\omega_{m}\right)|0\rangle$, which lives in the (reducible) tensor product space $\mathbb{H}^{(m)}=\mathbb{H}_{1}^{(1)} \otimes \mathbb{H}_{2}^{(1)} \ldots \otimes \mathbb{H}_{m}^{(1)}$. The first lemma deals with the weight of this state.

Lemma 1: The $\mathrm{SU}(m)$ weight of $\left|\Psi_{123 \ldots m}\right\rangle$ is [0].

Proof: This is immediate since every mode is occupied once, so $n_{i}=1 \forall i$. Since the component $k$ of the weight is $n_{k}-n_{k+1}, \hat{h}_{i}\left|\Psi_{123 \ldots m}\right\rangle=0 \forall i$.

From Lemma 1, we write $\left|\Psi_{123 \ldots m}\right\rangle$ as an expansion over zero-weight states in all irrep occurring in $\mathbb{H}^{(m)}$ according to

$$
\left|\Psi_{123 \ldots m}\right\rangle=\sum_{\alpha \lambda \ell} \tilde{c}_{\ell}^{(\lambda)_{\alpha}}\left|\psi_{\ell}^{(\lambda)_{\alpha}}\right\rangle, \quad \tilde{c}_{\ell}^{(\lambda)_{\alpha}}=\left\langle\psi_{\ell}^{(\lambda)_{\alpha}} \mid \Psi_{123 \ldots m}\right\rangle
$$

where $(\lambda)_{\alpha}$ is the $\alpha$ 'th copy of the irrep $(\lambda)_{\alpha}$ of $\mathrm{SU}(m)$ and $\ell$ labels those basis states that have 0 -weight in the irrep $(\lambda)_{\alpha}$ of $\mathrm{SU}(m)$.

Lemma 2: With the notation above:

$$
\sum_{\sigma} \chi^{\{\tau\}}(\sigma) P(\sigma)\left|\Psi_{123 \ldots m}\right\rangle=\frac{m !}{\operatorname{dim}(\tau)} \sum_{\alpha t} \tilde{c}_{t}^{(\tau)_{\alpha}}\left|\psi_{t}^{(\tau)_{\alpha}}\right\rangle .
$$

Proof: The proof of Lemma 2 relies on the duality between representations of the symmetric and the unitary groups. From duality, the basis states $\left\{\left|\psi_{t}^{(\tau)_{\alpha}}\right\rangle\right\}$ are also 
basis states for the irrep $\{\tau\}$ of $S_{m}$. Hence, using Eq. (8) we obtain

$$
\begin{aligned}
P(\sigma)\left|\Psi_{123 \ldots m}\right\rangle & =\sum_{\alpha \lambda \ell}\left|\psi_{\ell}^{(\lambda)_{\alpha}}\right\rangle\left\langle\psi_{\ell}^{(\lambda)_{\alpha}}|P(\sigma)| \Psi_{123 \ldots m}\right\rangle, \\
& =\sum_{\alpha \ell \lambda k}\left|\psi_{\ell}^{(\lambda)_{\alpha}}\right\rangle \Gamma_{\ell k}^{\{\lambda\}}(\sigma)\left\langle\psi_{k}^{(\lambda)_{\alpha}} \mid \Psi_{123 \ldots m}\right\rangle, \\
& =\sum_{\alpha \ell \lambda k}\left|\psi_{\ell}^{(\lambda)_{\alpha}}\right\rangle \Gamma_{k \ell}^{\{\lambda\}}\left(\sigma^{-1}\right) \tilde{c}_{k}^{(\lambda)_{\alpha}},
\end{aligned}
$$

where $\Gamma^{\{\lambda\}}$ is the unitary irrep $\{\lambda\}$ of $S_{m}$. Writing $\chi^{\{\tau\}}(\sigma)=\sum_{t} \Gamma_{t t}^{\{\tau\}}(\sigma)$ gives us

$$
\begin{aligned}
\sum_{\sigma} \chi^{\{\tau\}}(\sigma) P(\sigma)\left|\Psi_{123 \ldots m}\right\rangle & =\sum_{\alpha k \lambda \ell} \tilde{c}_{\ell}^{(\lambda)_{\alpha}}\left|\psi_{\ell}^{(\lambda)_{\alpha}}\right\rangle\left[\sum_{\sigma t} \Gamma_{t t}^{\{\tau\}}(\sigma) \Gamma_{\ell k}^{\{\lambda\}}\left(\sigma^{-1}\right)\right] \\
& =\frac{m !}{\operatorname{dim}(\tau)} \sum_{\alpha t} \tilde{c}_{t}^{(\tau)_{\alpha}}\left|\psi_{t}^{(\tau)_{\alpha}}\right\rangle
\end{aligned}
$$

where we have used the orthogonality of characters to arrive at Eq. (22).

Because the action of $\Omega \in \mathrm{SU}(m)$ commutes with the action of $\sigma \in S_{m}$, we have

$$
\begin{aligned}
\operatorname{Imm}^{\{\tau\}}(T(\Omega)) & =\sum_{\sigma} \chi^{\{\tau\}}(\sigma) P(\sigma)\left[T_{11}(\Omega) T_{22}(\Omega) \ldots T_{m m}(\Omega)\right], \\
& =\left\langle\Psi_{123 \ldots m}\right|[T(\Omega) \otimes T(\Omega) \ldots \otimes T(\Omega)] \\
& {\left[\sum_{\sigma} \chi^{\{\tau\}}(\sigma) P(\sigma)\right]\left|\Psi_{123 \ldots m}\right\rangle, } \\
& =\sum_{\alpha r t}\left(\tilde{c}_{r}^{\tau_{\alpha}}\right)^{*} \tilde{c}_{t}^{\tau_{\alpha}} \frac{m !}{\operatorname{dim}(\tau)} D_{r t}^{(\tau)}(\Omega) .
\end{aligned}
$$

Introducing the scaled coefficients $c_{t}^{(\tau)_{\alpha}}=\tilde{c}_{t}^{(\tau)_{\alpha}} \sqrt{\frac{m !}{\operatorname{dim}(\tau)}}$, we finally obtain

$$
\operatorname{Imm}^{\{\tau\}}(T(\Omega))=\sum_{r t}\left[\sum_{\alpha}\left(c_{r}^{(\tau)_{\alpha}}\right)^{*} c_{t}^{(\tau)_{\alpha}}\right] D_{r t}^{(\tau)}(\Omega),
$$

where the sums over $t$ and $r$ is a sum over zero-weight states in $(\tau)_{\alpha}$.

This result is not unexpected as the operator

$$
\hat{\Pi}^{\{\tau\}}=\left[\sum_{\sigma} \chi^{\{\tau\}}(\sigma) P(\sigma)\right], \quad \sigma \in S_{m}
$$

is a projector to that subspace of $S_{m}$ which has permutation symmetry $\{\tau\}$, and hence (by duality) is a projector to a subspace that carries (possibly multiple copies of) the irrep $(\tau)$ of $\mathrm{SU}(m)$ in the $m$-fold product $(1)^{\otimes m}$.

Theorem 3: (Kostant [2]) $\operatorname{Imm}^{\{\tau\}}(T(\Omega))=\sum_{t} D_{t t}^{(\tau)}(\Omega)$.

Proof: We present a proof that will eventually allow us to dispense with the requirements that $N=m$ and that states have zero-weight. Construct the matrix

$$
W_{r t}^{\{\tau\}}=\sum_{\alpha} c_{\alpha t}^{(\tau)}\left(c_{\alpha r}^{(\tau)}\right)^{*}
$$


Eq. (26) then becomes

$$
\operatorname{Imm}^{\{\tau\}}(T(\Omega))=\sum_{r t} W_{r t}^{\{\tau\}} D_{r t}^{(\tau)}(\Omega)=\operatorname{Tr}\left[W^{\{\tau\}} \mathcal{D}^{(\tau)}(\Omega)\right]
$$

with $\mathcal{D}^{(\tau)}(\Omega)$ defined in Eq. (3). Our objective is to prove that $W^{\{\tau\}}$ is the unit matrix.

Any immanant has the property of invariance under conjugation by elements in $S_{m}$ i.e., the immanant of any matrix satisfies

$$
\begin{aligned}
\operatorname{Imm}^{\{\tau\}}(T(\Omega)) & =\sum_{\sigma} \chi^{\{\tau\}}(\sigma) P(\sigma)\left[T_{11}(\Omega) T_{22}(\Omega) \ldots T_{m m}(\Omega)\right] \\
& =\sum_{\sigma} \chi^{\{\tau\}}(\sigma) P^{-1}(\bar{\sigma}) P(\sigma) P(\bar{\sigma})\left[T_{11}(\Omega) T_{22}(\Omega) \ldots T_{m m}(\Omega)\right]
\end{aligned}
$$

with $\sigma, \bar{\sigma} \in S_{m}$. Under conjugation by $\bar{\sigma}$, Eq. (26) becomes

$$
\begin{aligned}
\operatorname{Imm}^{\{\tau\}}(T(\Omega)) & =\operatorname{Tr}\left[\Gamma^{\{\tau\}}(\bar{\sigma}) W^{\{\tau\}} \Gamma^{\{\tau\}}\left(\bar{\sigma}^{-1}\right) \mathcal{D}^{(\tau)}(\Omega)\right] \\
& =\operatorname{Tr}\left[W^{\{\tau\}} \mathcal{D}^{(\tau)}(\Omega)\right]
\end{aligned}
$$

Since $\mathcal{D}^{(\tau)}(\Omega)$ is certainly not the unit matrix for arbitrary $\Omega$, it follows that

$$
\Gamma^{\{\tau\}}(\bar{\sigma}) W^{\{\tau\}} \Gamma^{\{\tau\}}\left(\bar{\sigma}^{-1}\right)=W^{\{\tau\}},
$$

i.e. the matrix $W^{\{\tau\}}$ is invariant under any permutation. By Schur's lemma $W^{\{\tau\}}$ must therefore be proportional to the unit matrix, i.e. we have $W_{t s}^{\{\tau\}}=\xi \delta_{t s}$ with $\xi$ the relevant constant of proportionality. The immanant thus takes the form

$$
\operatorname{Imm}^{\{\tau\}}(T(\Omega))=\xi\left(\sum_{t} D_{t t}^{(\tau)}(\Omega)\right) .
$$

To determine $\xi$, choose $\Omega=\mathbb{1}$. Then $T(\mathbb{1})$ is the $m \times m$ unit matrix, and $T_{k, \sigma(k)}(\mathbb{1})$ is zero unless $\sigma=\mathbb{1} \in S_{m}$. The immanant for $\Omega=\mathbb{1}$ is then just the dimension of the irrep $\{\tau\}$ and we have

$$
\operatorname{Imm}^{\{\tau\}}(T(\mathbb{1}))=\chi^{\{\tau\}}(\mathbb{1})=\operatorname{dim}(\tau), \quad \xi=\left(\sum_{t} 1\right)=\xi \operatorname{dim}(\{\tau\})
$$

since $D_{t t}^{(\tau)}(\mathbb{1})=1$. Hence, $\xi=1$ and the theorem is proved.

\section{Results on submatrices: the case $N<m$.}

We now consider the submatrices of $T$. In multiphoton interferometry, such submatrices describe the unitary scattering from an input state of the form

$$
\left|\Psi_{k_{1} \ldots k_{p}}\right\rangle=a_{k_{1}}^{\dagger}\left(\omega_{1}\right) a_{k_{2}}^{\dagger}\left(\omega_{2}\right) \ldots a_{k_{p}}^{\dagger}\left(\omega_{p}\right)|0\rangle, \quad p<m
$$

to an output state $\left|\Psi_{\ell_{1} \ldots \ell_{p}}\right\rangle$, which need not the identical to $\left|\Psi_{k_{1} \ldots k_{p}}\right\rangle$. Both input and output live in the reducible Hilbert space $\mathbb{H}^{(p)}$, and have expansions of the form

$$
\left|\Psi_{k_{1} \ldots k_{p}}\right\rangle=\sum_{\alpha \lambda \ell} \tilde{d}_{\ell}^{(\lambda)_{\alpha}}\left|\psi_{\ell}^{(\lambda)_{\alpha}}\right\rangle
$$

where $\left|\psi_{\ell}^{(\lambda)_{\alpha}}\right\rangle$ has weight $\left[k_{1}-k_{2}, k_{2}-k_{3}, \ldots, k_{p-1}-k_{p}\right]$. 


\subsection{Principal coaxial submatrices}

First we select from $T(\Omega)$ a principal submatrix $\bar{T}(\Omega)_{k}$, i.e., $\bar{T}(\Omega)_{k}$ is obtained by keeping rows and columns $k=\left(k_{1}, k_{2}, \ldots, k_{p}\right)$ with $p<m$. In such a case, the input and output states are identical. The permutation group $S_{p}$ shuffles the $p$ indices $k_{1}, k_{2}, \ldots, k_{p}$ amongst themselves. Although the submatrix $\bar{T}(\Omega)_{k}$ is not unitary, the proof of Theorem 3 does not depend on the unitarity of $T(\Omega)$ and so can be copied to show

Corollary 4: The immanant $\operatorname{Imm}_{k}^{\{\lambda\}}(T(\Omega))$ of a submatrix $\bar{T}(\Omega)_{k}$, which is a principal submatrix of $T$, is given by

$$
\operatorname{Imm}_{k}^{\{\lambda\}}(T(\Omega))=\sum_{r} D_{r r}^{(\lambda)}(\Omega)
$$

where $(\lambda)$ is the irrep of $\mathrm{SU}(m)$ corresponding to the partition $\{\lambda\}$, and where the sum over $r$ is a sum over all the states in $(\lambda)$ with weight $\left[k_{1}-k_{2}, k_{2}-k_{3}, \ldots, k_{p-1}-k_{p}\right]$; following Eq.(36) this is the weight of $\left|\Psi_{k_{1} \ldots k_{p}}\right\rangle$ in Eq. (35) and need not be zero.

As an example, one can verify that, if we strip the $5 \times 5$ fundamental matrix representation of $\mathrm{SU}(5)$ from its third and fifth rows and columns, then the states entering in the sum of Eq. (37) are linear combinations of terms of the form

$$
P(\sigma)\left[a_{1}^{\dagger}\left(\omega_{1}\right) a_{2}^{\dagger}\left(\omega_{2}\right) a_{4}^{\dagger}\left(\omega_{3}\right)|0\rangle\right]
$$

with weight $[0,1,-1,1]$. Using the $\operatorname{su}(k) \downarrow \mathrm{su}(k-1)$ branching rules $[16,17]$ to label basis states, the $\{2,1\}$ immanant of this submatrix is the sum

$$
\begin{aligned}
\operatorname{Imm}_{124}^{\{2,1\}}(T(\Omega))= & D_{11010(2)(1)\left(\frac{1}{2}\right) ; 11010(2)(1)\left(\frac{1}{2}\right)}^{(1,1)}(\Omega) \\
& +D_{11010(0,1)(1)\left(\frac{1}{2}\right) ; 11010(0,1)(1)\left(\frac{1}{2}\right)}^{(1,1)}(\Omega),
\end{aligned}
$$

where the labels $(2)(1)\left(\frac{1}{2}\right)$ and $(0,1)(1)\left(\frac{1}{2}\right)$ refer to the $\mathrm{su}(4) \supset \mathrm{su}(3) \supset \mathrm{su}(2)$ chains of irreps (recall that trailing 0 s are omitted).

Littlewood [1] has established a number of relations between immanants of a matrix and sums of products of immanants of principal coaxial submatrices. For instance, the equality for Schur functions $\{3\}\{1\}=\{3,1\}+\{4\}$ yields the immanant relation

$$
\begin{aligned}
\sum_{i j k \ell}\left(\operatorname{Imm}_{i j k}^{\{3\}}(T(\Omega))\right)\left(\operatorname{Imm}_{\ell}^{\{1\}}(T(\Omega))\right) & \\
= & \operatorname{Imm}^{\{3,1\}}(T(\Omega))+\operatorname{Imm}^{\{4\}}(T(\Omega))
\end{aligned}
$$

where the sum over $i j k \ell$ is a sum over complementary coaxial submatrices, i.e.

\begin{tabular}{ll||ll}
$i j k$ & $\ell$ & $i j k$ & $\ell$ \\
\hline 123 & 4 & 124 & 3 \\
134 & 2 & 234 & 1
\end{tabular}

This expands to a sum of products of immanants of submatrices given explicitly by

$$
\begin{aligned}
& \left(\operatorname{Imm}_{123}^{\{3\}}(T(\Omega))\right)\left(\operatorname{Imm}_{4}^{\{1\}}(T(\Omega))\right)+\left(\operatorname{Imm}_{124}^{\{3\}}(T(\Omega))\left(\operatorname{Imm}_{3}^{\{1\}}(T(\Omega))\right)\right. \\
& +\left(\operatorname{Imm}_{134}^{\{3\}}(T(\Omega))\right)\left(\operatorname{Imm}_{2}^{\{1\}}(T(\Omega))\right)+\left(\operatorname{Imm}_{234}^{\{3\}}(T(\Omega))\right)\left(\operatorname{Imm}_{1}^{\{1\}}(T(\Omega))\right) \\
& =\operatorname{Imm}^{\{3,1\}}(T(\Omega))+\operatorname{Imm}^{\{4\}}(T(\Omega)),
\end{aligned}
$$


which becomes an equality on the corresponding products of sum of SU(4) D-functions:

$$
\begin{aligned}
& D_{1110(2)\left(\frac{1}{2}\right) ; 1110(2)\left(\frac{1}{2}\right)}^{(3)}(\Omega) D_{0001(1)\left(\frac{1}{2}\right) ; 0001(1)\left(\frac{1}{2}\right)}^{(1)}(\Omega) \\
& \quad+D_{1101(2)\left(\frac{1}{2}\right) ; 1101(2)\left(\frac{1}{2}\right)}^{(3)}(\Omega) D_{0010(1)\left(\frac{1}{2}\right) ; 0010(1)\left(\frac{1}{2}\right)}^{(1)}(\Omega) \\
& \quad+D_{1011(2)(1) ; 1011(2)(1)}^{(3)}(\Omega) D_{0100(1)(0) ; 0100(1)(0)}^{(1)}(\Omega) \\
& \quad+D_{0111(3)(1) ; 0111(3)(1)}^{(3)}(\Omega) D_{1000(0)(0) ; 1000(0)(0)}^{(1)}(\Omega) \\
& =D_{1111(3)(1) ; 1111(3)(1)}^{(21)}(\Omega)+D_{1111(11)(1) ; 1111(11)(1)}^{(21)}(\Omega) \\
& \quad+D_{1111(11)(0) ; 1111(11)(0)}^{(21)}(\Omega)+D_{1111(3)(1) ; 1111(3)(1)}^{(4)}(\Omega) .
\end{aligned}
$$

The subgroup labels are obtained by systematically using the $\mathrm{su}(k) \downarrow \mathrm{su}(k-1)$ branching rules [18].

\subsection{Generic submatrices}

To fix ideas, we start with the $4 \times 4$ matrix $T$ and remove row 1 and column 2 to obtain the submatrix $\bar{T}$ :

$$
T(\Omega) \rightarrow \bar{T}(\Omega)=\left(\begin{array}{lll}
T_{21}(\Omega) & T_{23}(\Omega) & T_{24}(\Omega) \\
T_{31}(\Omega) & T_{33}(\Omega) & T_{34}(\Omega) \\
T_{41}(\Omega) & T_{43}(\Omega) & T_{44}(\Omega)
\end{array}\right)
$$

The immanants of $3 \times 3$ submatrix $\bar{T}(\Omega)$ are in the form

$$
\begin{aligned}
\operatorname{Imm}^{\{\lambda\}}(\bar{T}(\Omega)) & =\sum_{\sigma} \chi^{\{\lambda\}}(\sigma) P(\sigma)\left[T_{11}(\Omega) T_{22}(\Omega) T_{34}(\Omega)\right] \\
& =\hat{\Pi}^{\{\lambda\}}\left[T_{11}(\Omega) T_{22}(\Omega) T_{34}(\Omega)\right]
\end{aligned}
$$

where $\hat{\Pi}^{\{\lambda\}}$ is the immanant projector of Eq. (27) and $\sigma$ permutes the triple (124).

Let $\left\{a_{k}^{\dagger}\left(\omega_{k}\right)|0\rangle, k=1, \ldots, 4\right\}$ be a basis for the fundamental irrep of $\mathrm{SU}(4)$, and define

$$
\begin{aligned}
& \left|\Psi_{134}\right\rangle:=a_{1}^{\dagger}\left(\omega_{1}\right) a_{3}^{\dagger}\left(\omega_{2}\right) a_{4}^{\dagger}\left(\omega_{3}\right)|0\rangle, \\
& \left|\Phi_{234}\right\rangle:=a_{2}^{\dagger}\left(\omega_{1}\right) a_{3}^{\dagger}\left(\omega_{2}\right) a_{4}^{\dagger}\left(\omega_{3}\right)|0\rangle
\end{aligned}
$$

as three-particle states elements of $\mathbb{H}^{\{1\} \otimes\{1\} \otimes\{1\}}$. Clearly there is $\sigma^{\prime} \in S_{4}$ such that

$$
\left|\Psi_{234}\right\rangle=P\left(\sigma^{\prime}\right)\left|\Phi_{134}\right\rangle \text {. }
$$

Indeed by inspection this element is given by $P\left(\sigma^{\prime}\right)=P_{12}$. More generally, if

$$
\begin{array}{ll}
\left|\Phi_{k}\right\rangle=a_{k_{1}}^{\dagger}(\omega) a_{k_{2}}^{\dagger}\left(\omega_{2}\right) a_{k_{3}}^{\dagger}\left(\omega_{3}\right)|0\rangle, & k=\left(k_{1}, k_{2}, k_{3}\right), \\
\left|\Psi_{q}\right\rangle=a_{q_{1}}^{\dagger}(\omega) a_{q_{2}}^{\dagger}\left(\omega_{2}\right) a_{q_{3}}^{\dagger}\left(\omega_{3}\right)|0\rangle, & q=\left(q_{1}, q_{2}, q_{3}\right),
\end{array}
$$

then there is $\sigma_{q k}$ exists such that $\left|\Psi_{q}\right\rangle=P\left(\sigma_{q k}\right)\left|\Phi_{k}\right\rangle$. As the action of the permutation group commutes with the action of the unitary group:

$$
\begin{aligned}
\operatorname{Imm}^{\{\lambda\}}(\bar{T}(\Omega))_{k q} & =\left\langle\Phi_{k}\left|[T(\Omega) \otimes T(\Omega) \ldots \otimes T(\Omega)] \hat{\Pi}^{\{\lambda\}} P\left(\sigma_{q k}\right)\right| \Phi_{k}\right\rangle \\
& =\left\langle\Phi_{k}\left|\hat{\Pi}^{\{\lambda\}}[T(\Omega) \otimes T(\Omega) \ldots \otimes T(\Omega)] P\left(\sigma_{q k}\right)\right| \Phi_{k}\right\rangle
\end{aligned}
$$




$$
\begin{aligned}
=\sum_{r s \alpha} & \left\langle\Phi_{k}\left|\hat{\Pi}^{\{\lambda\}}\right| \psi_{r}^{(\lambda)_{\alpha}}\right\rangle \\
& \times\left\langle\psi_{r}^{(\lambda)_{\alpha}}\left|T^{(\lambda)}(\Omega) P\left(\sigma_{q k}\right)\right| \psi_{s}^{(\lambda)_{\alpha}}\right\rangle\left\langle\psi_{s}^{(\lambda)_{\alpha}} \mid \Phi_{k}\right\rangle
\end{aligned}
$$

Now, the permutation $P\left(\sigma_{q k}\right)$ is represented by a unitary matrix in the carrier space $(\lambda)_{\alpha}$. Thus, there exist $\Omega_{q k} \in \mathrm{SU}(4)$ and a phase $\zeta$ such that $P\left(\sigma_{q k}\right)\left|\psi_{s}^{(\lambda)_{\alpha}}\right\rangle=e^{i \zeta} T\left(\Omega_{q k}\right)\left|\psi_{s}^{(\lambda)_{\alpha}}\right\rangle$. This transforms our original problem back to the case of principal submatrices, but with now an element $\Omega \cdot \Omega_{q k}$ i.e.,

$$
\operatorname{Imm}^{\{\lambda\}}(\bar{T}(\Omega))=\sum_{t} D_{t t}^{(\lambda)}\left(\Omega \cdot \Omega_{q k}\right) .
$$

Unfortunately, the action $P\left(\sigma_{q k}\right)\left|\psi_{s}^{(\lambda)_{\alpha}}\right\rangle$ is in general highly non-trivial [19-21] and it is not obvious how to find $\Omega_{q k}$, much less $\left(\Omega \cdot \Omega_{q k}\right)$. Nevertheless, we found that the sum of $D$-functions that occur on the right hand side of Eq. (53) always contains the same number of $D$ as the dimension of the dual irrep $\{\tau\}$, and that the coefficients of these $D$ 's is always one. This result relied on (i) evaluating the appropriate group functions using the algorithm [18], (ii) explicitly constructing each of the immanants of all possible $4 \times 4$ submatrices and of all possible $3 \times 3$ submatrices of the fundamental irrep of $\mathrm{SU}(5)$ and (iii) explicitly constructing the immanants of $3 \times 3$ submatrices of the fundamental irrep of $\mathrm{SU}(4)$ or $\mathrm{SU}(5)$.

Thus, in the specific case of the submatrix given in Eq. (44), we have

$$
\operatorname{Imm}^{\{21\}}(\bar{T}(\Omega))_{(234)(134)}=D_{0111(2)\left(\frac{1}{2}\right) ; 1011(2)\left(\frac{1}{2}\right)}^{(1,1)}(\Omega)+D_{0111(11)\left(\frac{1}{2}\right) ; 1011(11)\left(\frac{1}{2}\right)}^{(1,1)}(\Omega) .
$$

We also verified that a similar identity holds for all $3 \times 3$ submatrices of $T(\Omega) \in \mathrm{SU}(4)$. For instance,

$$
\begin{aligned}
& \operatorname{Imm}^{\{21\}}(\bar{T}(\Omega))_{(234)(124)}=D_{0111,(2)\left(\frac{1}{2}\right) ; 1101(2)\left(\frac{1}{2}\right)}^{(1,1)}(\Omega)+D_{0111(11)\left(\frac{1}{2}\right) ; 1101(01)\left(\frac{1}{2}\right)}^{(1,1)}(\Omega),(56) \\
& \operatorname{Imm}^{\{21\}}(\bar{T}(\Omega))_{(134)(124)}=D_{1011(2)\left(\frac{1}{2}\right) ; 1110(2)\left(\frac{1}{2}\right)}^{(1,1)}(\Omega)+D_{1011(11)\left(\frac{1}{2}\right) ; 1110(01)\left(\frac{1}{2}\right)}^{(1,1)}(\Omega)
\end{aligned}
$$

Likewise, we have, for $T(\Omega) \in \mathrm{SU}(5)$,

$$
\begin{aligned}
& \operatorname{Imm}^{\{21\}}(\bar{T}(\Omega))_{(1345)(1235)} \\
& \quad=D_{01101(11)(2)\left(\frac{1}{2}\right), 10110(2)(2)\left(\frac{1}{2}\right)}^{(1,1)}(\Omega)+D_{01101(11)(01)\left(\frac{1}{2}\right), 10110(01)(01)\left(\frac{1}{2}\right)}^{(1,1)}(\Omega),(58) \\
& \quad \operatorname{Imm}^{\{31\}}(\bar{T}(\Omega))_{(235)(134)}=D_{10111(3)(3)(1), 11101(3)(2) \frac{1}{2}}^{(2,1)}(\Omega) \\
& \quad+D_{10111(11)(11)(1), 11101(11)(2) \frac{1}{2}}^{2,1}(\Omega)+D_{10111(11)(11)(0), 11101(11)(01) \frac{1}{2}}^{(2,1)}(\Omega)
\end{aligned}
$$

this last being an example of a $4 \times 4$ submatrix not principal coaxial. We thus conjecture that, even for generic submatrices, $\operatorname{Imm}^{\{\lambda\}}(\bar{T}(\Omega))_{k q}$ is a sum of $\operatorname{dim}\{\lambda\}$ distinct $D$ 's with coefficients equal to +1 , although we cannot yet formulate a solid proof.

\section{Outer plethysms and subgroups}

We now consider an application of our result to immanants of unitary matrices that are not in the fundamental representation. The difficulty in this case is that the various 
$S_{N}$-invariant subspaces in the $N$-fold tensor product no longer contain a single $\mathrm{SU}(m)$ irrep. Consider for instance $T(\Omega)$, the $4 \times 4(J=3 / 2)$ matrix representation of $\mathrm{SU}(2)$. Using the standard $D_{M^{\prime} M}^{J}(\Omega)$ notation for the group functions and $|J M\rangle$ as the notation for basis states, we have

$$
\operatorname{Imm}^{\{2,2\}}(T(\Omega))=\frac{26}{35} D_{00}^{4}(\Omega)+\frac{6}{7} D_{00}^{2}(\Omega)+\frac{2}{5} D_{00}^{0}(\Omega),
$$

which is still a sum of diagonal group functions $\sum_{J} c_{J} D_{00}^{J}(\Omega)$. The dimension of the $S_{4}$ irrep $\{2,2\}$ is 2 and, while the sum contains more than two terms, we still have $\sum_{J} c_{J}=\operatorname{dim}(\{2,2\})$. The possible values of $J$ entering in the sum are those that occur in the (outer) plethysm $(3 / 2) \otimes_{\wp}\{2,2\}$, and can be found using Schur function techniques. (Here, $\otimes_{\wp}$ denotes the plethysm operation.)

A more sophisticated application is to the evaluation of immanants of the $6 \times 6$ matrix representation $(2,0)$ of $\mathrm{SU}(3) .(2)^{\otimes 6}$ contains more than one $\mathrm{SU}(3)$ irrep. For instance, one might consider various immanants of the $6 \times 6$ matrix $T(\Omega)$ of the $(2,0)$ of $\mathrm{SU}(3)$. We may consider this matrix as an element of the $\mathrm{SU}(3)$ subgroup of $\mathrm{SU}(6)$.

As an example, the permanant $\operatorname{Imm}^{\{6\}}(T(\Omega))=\left\langle\Psi_{444}|R(\Omega)| \Psi_{444}\right\rangle$ but the state $\left|\Psi_{444}\right\rangle$ is now a linear combination of $\mathrm{SU}(3)$ states in various irreps that occur in $(2) \otimes_{\wp}\{6\}$. Explicitly, we have

$$
\begin{aligned}
\left|\Psi_{444}\right\rangle & =\sqrt{\frac{64}{385}}|(12) 444(4)\rangle+\sqrt{\frac{18}{385}}|(8,2) 444(\alpha)\rangle+\sqrt{\frac{4}{21}}|(4,4) 444(\beta)\rangle \\
& +\frac{1}{3}|(6) 222(2)\rangle+\sqrt{\frac{16}{63}}|(0,6) 444(2)\rangle+\sqrt{\frac{2}{45}}|(0) 000(0)\rangle,
\end{aligned}
$$

where

$$
\begin{gathered}
|(8,2) 444(\alpha)\rangle=\sqrt{\frac{10}{21}}|(8,2) 444(4)\rangle+\sqrt{\frac{11}{21}}|(8,2) 444(2)\rangle \\
|(4,4) 444(\beta)\rangle=\sqrt{\frac{12}{35}}|(4,4) 444(4)\rangle+\sqrt{\frac{4}{21}}|(4,4) 444(2)\rangle \\
+\sqrt{\frac{7}{15}}|(4,4) 444(0)\rangle .
\end{gathered}
$$

Note that both $|(8,2) 444(\alpha)\rangle$ and $|(4,4) 444(\beta)\rangle$ carry the fully symmetric $\{3\}$ irrep of $S_{3}$, as can be verified using the matrix elements of the permutation operators given in [21]. The permanent $\operatorname{Imm}^{\{6\}}(T(\Omega))$ can be written as a sum of diagonal $D$-functions:

$$
\begin{aligned}
& \operatorname{Imm}^{\{6\}}(T(\Omega)) \\
& =c_{1} D_{(444 ; 4)(444 ; 4)}^{(12,0)} \\
& \quad+c_{2} D_{(444 ; 4)(444 ; 4)}^{(8,2)}+c_{3} D_{(444 ; 4)(444 ; 2)}^{(8,2)}+c_{4} D_{(444 ; 2)(444 ; 4)}^{(8,2)}+c_{5} D_{(444 ; 2)(444 ; 2)}^{(8,2)} \\
& \quad+c_{6} D_{(444 ; 4)(444 ; 4)}^{(4,4)}+c_{7} D_{(444 ; 4)(444 ; 2)}^{(4,4)}+c_{8} D_{(444 ; 4)(444 ; 0)}^{(4,4)}+c_{9} D_{(444 ; 2)(444 ; 4)}^{(4,4)} \\
& \quad+c_{10} D_{(444 ; 2)(444 ; 2)}^{(4,4)}+c_{11} D_{(444 ; 2)(444 ; 0)}^{(4,4)}+c_{12} D_{(444 ; 0)(444 ; 4)}^{(4,4)}+c_{13} D_{(444 ; 0)(444 ; 2)}^{(4,4)} \\
& \quad+c_{14} D_{(444 ; 0)(444 ; 0)}^{(4,4)} \\
& \quad+c_{15} D_{(222 ; 2)(222 ; 2)}^{(6,0)}+c_{16} D_{(444 ; 2)(444 ; 2)}^{(0,6)}+c_{17} D_{(000 ; 0)(000 ; 0)}^{(0,0)}
\end{aligned}
$$


with solution

\begin{tabular}{|l|l|l|l|l|}
\hline$c_{1}=\frac{64}{385}$ & & & & \\
\hline$c_{2}=\frac{60}{539}$ & $c_{3}=\frac{6}{49} \sqrt{\frac{10}{11}}$ & $c_{4}=\frac{6}{49} \sqrt{\frac{10}{11}}$ & $c_{5}=\frac{6}{49}$ & \\
\hline$c_{6}=\frac{16}{245}$ & $c_{7}=\frac{16 \sqrt{5}}{147}$ & $c_{8}=\frac{8}{105}$ & $c_{9}=\frac{16 \sqrt{5}}{147}$ & \\
\hline$c_{10}=\frac{16}{441}$ & $c_{11}=\frac{8}{63 \sqrt{5}}$ & $c_{12}=\frac{8}{105}$ & $c_{13}=\frac{8}{63 \sqrt{5}}$ & $c_{14}=\frac{4}{45}$ \\
\hline$c_{15}=\frac{1}{9}$ & $c_{16}=\frac{16}{63}$ & $c_{17}=\frac{2}{45}$ & & \\
\hline
\end{tabular}

The coefficients $c_{k}$ are immediately seen to be related to coefficents in Eqs.(61)(63). As anticipated from the theorem, the sum of coefficients of diagonal $D$ s $c_{1}+c_{2}+c_{5}+c_{6}+c_{10}+c_{14}+c_{15}+c_{16}+c_{17}=1$.

\section{Discussion and conclusion}

The relation between characters of $S_{N}$ and $\mathrm{U}(n)$ is well known and a rich source of results in mathematical physics. Our work expands these beyond characters to novel connections between immanants and the group functions proper. Results on group functions (see [21,22] as well as [18] and references therein) are comparatively less common than those available for, say, the calculation of generator matrix elements or Clebsch-Gordan coefficients. We hope some of the results given here might be useful in providing impetus or remedy to this relative paucity of results on group functions.

Immanants are connected to the interferometry of partially distinguishable pulses [4-6]; the associated permutation symmetries lead to novel interpretations of immanants as a type of normal coordinates describing lossless passive interferometers [6]. This connection immediately provides a physical interpretation to the appropriate combinations of group functions corresponding to these immanants, and should stimulate further development of toolkits to compute group functions.

Conjectures in complexity theory regarding the behaviour of permanents of large unitary matrices may also provide an entry point towards understanding the behaviour of $D$-functions in similar asymptotic regimes. It remains to see if this line of thought can also be turned around: it might be possible to use results on the asymptotic behaviour of $D$-functions to establish some conjectures on the behaviour of immanants of large matrices.

Finally, although the Schur-Weyl duality is not directly applicable to subgroups of the unitary groups, the permutation group retains its deep connection with representations of the classical groups, which are considered as subgroups of the unitary groups $[23,24]$. Hence, it might be possible to extend the results of this paper to functions of the orthogonal or symplectic groups, thus generalizing the result of Sec. 5 on immanants associated with plethysms of representations. 


\section{Acknowledgements}

This work was supported by NSERC and Lakehead University. ID acknowledges AITF, NSERC and USARO for financial support.

\section{References}

[1] Littlewood D 1950 The Theory of Group Characters and Matrix Representations of Groups AMS Chelsea Publishing Series (Gloucestershire: Clarendon Press) ISBN 9780821874356

[2] Kostant B 1995 J. Amer. Math. Soc. 8 181-186 ISSN 08940347 URL http://www . jstor.org/stable/2152885

[3] Minc H 1984 Permanents vol 6 (Cambridge: Cambridge University Press)

[4] Tan S H, Gao Y Y, de Guise H and Sanders B C 2013 Phys. Rev. Lett. 110113603 ISSN 0031-9007 URL http://dx.doi.org/10.1103/PhysRevLett.110.113603

[5] de Guise H, Tan S H, Poulin I P and Sanders B C 2014 Phys. Rev. A. 89 ISSN 1094-1622 URL http://dx.doi.org/10.1103/physreva.89.063819

[6] Tillmann M, Tan S H, Stoeckl S E, Sanders B C, de Guise H, Heilmann R, Nolte S, Szameit A and Walther P 2015 Phys. Rev. X 5(4) 041015 URL http://link.aps.org/doi/10.1103/PhysRevX.5.041015

[7] Shchesnovich V S 2015 Phys. Rev. A 91 ISSN 1094-1622 URL http://dx.doi.org/10.1103/PhysRevA.91.013844

[8] Tichy M $\mathrm{C} \quad 2015$ Phys. Rev. A $\mathbf{9 1}$ ISSN 1094-1622 URL http://dx.doi.org/10.1103/PhysRevA.91.022316

[9] Aaronson S and Arkhipov A 2013 Theory Comput. 9 143-252 URL http://dx.doi.org/10.4086/toc.2013.v009a004

[10] Aaronson S and Arkhipov A 2014 Quantum Info. Comput. 14 1383-1423 ISSN 1533-7146 URL http: //dl . acm.org/citation. cfm?id=2685179. 2685186

[11] Pate T H 1992 Linear Algebra Appl. 162-164 639-650 ISSN 0024-3795 URL http://dx.doi .org/10.1016/0024-3795(92) 90398-T

[12] Pate $\mathrm{T}$ H 1994 Linear Algebra Appl. 212-213 $31-44$ ISSN $0024-3795$ URL http://dx.doi.org/10.1016/0024-3795(94)90395-6

[13] Rhoades B and Skandera M 2005 Ann. Comb. 9 $451-494$ URL http://dx.doi.org/10.1007/s00026-005-0268-0

[14] Weyl H 1950 The Theory of Groups and Quantum Mechanics (Mineola: Dover Publications Inc.)

[15] Rowe D J, Carvalho M J and Repka J 2012 Rev. Mod. Phys. 84 711-757 ISSN 1539-0756 URL http://dx.doi.org/10.1103/RevModPhys.84.711

[16] Slansky R 1981 Phys. Rep. 79 1-128 URL http://dx.doi.org/10.1016/0370-1573(81) 90092-2

[17] Whippman M L 1965 J. Math. Phys. 6 1534-1539 URL http://dx.doi.org/10.1063/1.1704691

[18] Dhand I, Sanders B C and de Guise H 2015 J. Math. Phys. 56 111705 URL http://scitation.aip.org/content/aip/journal/jmp/56/11/10.1063/1.4935433

[19] Kramer $\mathrm{P}$ and Moshinsky M 1966 Nucl. Phys. 82 241-274 ISSN 0029-5582 URL http://dx.doi.org/10.1016/0029-5582(66)90001-0

[20] Moshinsky M and Chacon E 1968 Racah coefficients and states with permutational symmetry Spectroscopic and Group Theoretical Methods in Physics ed Giovannini A and Smith D A (Amsterdam: North-Holland Publishing Co.)

[21] Rowe D J, Sanders B C and de Guise H 1999 J. Math. Phys. 403604 ISSN 0022-2488 URL http://dx.doi.org/10.1063/1.532911

[22] Louck J D 1970 Am. J. Phys. 38 3-42 URL http://dx.doi.org/10.1119/1.1976225

[23] Butler P H and Wybourne B G 1969 J. Phys. France 30 795-802 URL http://dx.doi.org/10.1051/jphys:019690030010079500 
[24] Wybourne B G 1974 Classical Groups for Physicists (New York: John Wiley and Sons, Inc.,) 